\title{
A TWO CARDINAL THEOREM FOR HOMOGENEOUS SETS AND THE ELIMINATION OF MALITZ QUANTIFIERS
}

\author{
BY
}

\author{
PHILIPP ROTHMALER AND PETER TUSCHIK
}

\begin{abstract}
Sufficient conditions for the eliminability of Malitz quantifiers in a complete first order theory are given. Proving that certain superstable and not $\omega$-stable theories satisfy these conditions, a question of Baldwin and Kueker is answered negatively.
\end{abstract}

Introduction. The aim of the present paper is to give a first approximation to the problem of finding natural conditions for a theory in the first order language to admit the elimination of certain Malitz quantifiers in the same sense as the substructure completeness theorem does for elimination of elementary quantifiers. In other words, we look for the classes of first order theories which remain essentially the same when we add certain Malitz quantifiers. Thus, the present paper is a contribution to first order model theory, and not to logics with additional quantifiers. Our investigations were inspired by the second author's mostly unpublished work (cf. [TU1] and [TU2]) concerning the eliminability of the quantifiers "there are $\aleph_{\alpha}$ many" (= Malitz quantifiers for 1-tuples) which we intended to extend to the general case. Although we did not completely succeed in our design, we obtained some partial results which we present in the following order.

$\$ 1$ contains definitions, conventions, central properties related to the quantifiers, and an eliminability condition for Ramsey quantifiers (= Malitz quantifiers in the $\boldsymbol{\aleph}_{0}$-interpretation). $\$ 2$ is devoted to a two cardinal theorem for maximally homogeneous sets (without any reference to quantifiers). $\S 3$ is an application of $\S 2$ to the eliminability problem. $\$ 4$ provides a negative answer to a question of Baldwin and Kueker [BK, Question 4]. Open questions are scattered about the paper. Our results were obtained independently of [BK] in September-October 1979. For further historical remarks, see below.

1. Preliminaries. In our notation we follow primarily [SH] with the following exceptions.

$A, B, \ldots, M, N$ are models and we do not distinguish between a model and its universe. $X, Y$ are sets; $u, v, x, z$ are variables; $\bar{u}, \bar{v}, \bar{x}$ are finite sequences (tuples) of variables. $y$ is reserved for an element of a homogeneous set. Denote by $l(\bar{z})$ the

Received by the editors January 2, 1980.

AMS (MOS) subject classifications (1970). Primary 02H05, 02B20, 02G15, 02G20.

Key words and phrases. Elimination of generalized quantifiers, first order theories, f.c.p., two cardinal theorem, stability, categoricity, finite axiomatizability. 
length of the sequence $\bar{z}$. Throughout the paper $T$ denotes a complete theory with infinite models in a countable first order language $L$. In $\S 4, T$ has a more specific meaning. We do not distinguish between symbols of $L$ and their interpretations in a given model, nor between elements and their names with one exception: For $X \subseteq A$ we write $\operatorname{Th}\left(\langle A, \underline{c}\rangle_{c \in X}\right)$ (in short $\operatorname{Th}(\langle A, X\rangle)$ ) to mark out that $\underline{c}$ is a new constant symbol which is the name of $c . A \vDash F(\bar{a})$ simply denotes that $\bar{a} \in A$ satisfies the formula $F(\bar{x})$ in $A$, where it is always assumed that $l(\bar{a})=l(\bar{x})$. We write $F(A)$ for the set $\{a \in A: A \vDash F(a)\}$. For $F(x)$ a 1-placed formula $\bar{F}(\bar{x})$ is an abbreviation of $\&_{i=1}^{m} F\left(x_{i}\right)$, where $\bar{x}=\left(x_{1}, \ldots, x_{m}\right)$.

We use also the following notion of restriction which can be found in the literature. If $F(\bar{x}), K(x)$ are formulae of $L, F^{K}(\bar{x})$, the restriction of $F$ to $K$, and $A \uparrow K(A)$, the restriction of $A$ to $K(A)$, are defined in such a way that for all $\bar{a} \in K(A), A \uparrow K(A) \vDash F(\bar{a})$ iff $A \vDash F^{K}(\bar{a})$. For $X$ a set of $L$-formulae, $X^{K}$ denotes the set $\left\{F^{K}: F \in X\right\}$.

A subset $Y$ of a model $A$ is said to be homogeneous for a formula $\varphi\left(x_{1}, \ldots, x_{m}\right)$ if $A \vDash \varphi\left(y_{1}, \ldots, y_{m}\right)$ for all $\left(y_{1}, \ldots, y_{m}\right)$ of $Y^{m}$. $Y$ is called maximally homogeneous for $\varphi$ in $A$ if $Y$ is homogeneous for $\varphi$ in $A$ and no proper superset of $Y$, which is also a subset of $A$, is homogeneous for $\varphi$ in $A$.

Let us now introduce the interpretations of the Malitz quantifiers (for syntactical and other details we refer to [MM]). We define $A \vDash Q_{\alpha}^{m} x_{1} \ldots x_{m} \varphi\left(x_{1}, \ldots, x_{m}\right)$ if there is a subset $Y$ in $A$ of cardinality $\boldsymbol{N}_{\alpha}$ which is homogeneous for $\varphi$ in $A$. For $\alpha=0$ the quantifiers are also called Ramsey quantifiers.

In [MM] there is considered in fact another interpretation connected with the following weaker notion of homogeneity. $Y$ is called weakly homogeneous for $\varphi$ in $A$ if $A \vDash\left(\&_{1 \leqslant i<j \leqslant m} y_{i} \neq y_{j}\right) \rightarrow \varphi\left(y_{1}, \ldots, y_{m}\right)$ for all $\left(y_{1}, \ldots, y_{m}\right) \in Y^{m}$. Denote $+Q^{m}$ the appropriate quantifier. Then it is easily seen that the following hold.

$$
\begin{aligned}
& A \vDash+ Q_{\alpha}^{m} x_{1} \ldots x_{m} \varphi\left(x_{1}, \ldots, x_{m}\right) \\
& \leftrightarrow Q_{\alpha}^{m} x_{1} \ldots x_{m}\left(\left(\underset{1<i<j<m}{\&} x_{i} \neq x_{j}\right) \rightarrow \varphi\left(x_{1}, \ldots, x_{m}\right)\right), \\
& A \vDash Q_{\alpha}^{m} x_{1} \ldots x_{m} \varphi\left(x_{1}, \ldots, x_{m}\right) \leftrightarrow+Q_{\alpha}^{m} x_{1} \ldots x_{m}(\underset{\bar{u} \in J}{\&} \varphi(\bar{u})),
\end{aligned}
$$

where $J$ is the set of all sequences of elements from $\left\{x_{1}, \ldots, x_{m}\right\}$ having length $m$. Thus, $Q_{\alpha}^{m}$ is eliminable iff $+Q_{\alpha}^{m}$ is eliminable. Notice, $Q_{\alpha}^{1}$ is the usual quantifier "there are $\boldsymbol{N}_{\alpha}$ many".

If $\varphi(\bar{x}, \bar{v}) \in L$, call the quantifier $Q_{\alpha}^{m} \varphi$-definable in $T$ if there is an $n$ such that $A \vDash \forall \bar{v}\left(H_{n}^{m} \bar{x} \varphi(\bar{x}, \bar{v}) \rightarrow Q_{\alpha}^{m} \bar{x} \varphi(\bar{x}, \bar{v})\right)$ for all models $A$ of $T$ of cardinality $\geqslant \boldsymbol{N}_{\alpha}$, where $H_{n}^{m} \bar{x} \varphi(\bar{x}, \bar{v})$ is the first order expression of "there is a set of cardinality $\geqslant n$ which is homogeneous for $\varphi$ ". Call the quantifier $Q_{\alpha}^{m} \varphi$-eliminable in $T$ if there is a first order formula $\psi(\bar{v})$ such that $A \vDash \forall \bar{v}\left(Q_{\alpha}^{m} \bar{x} \varphi(\bar{x}, \bar{v}) \leftrightarrow \psi(\bar{v})\right)$ for all models $A$ of $T$ of cardinality $\geqslant \boldsymbol{\aleph}_{\alpha}$.

The following observation is basic for elimination results.

LEMMA. The quantifier $Q_{\alpha}^{m}$ is $\varphi$-eliminable in $T$ iff it is $\varphi$-definable in $T$. 
For $\alpha=0$ this is Theorem 1 of [BK]. However, the second author was familiar with various versions of it. In fact the lemma holds for every quantifier we deal with in the present paper (mutatis mutandis in the sentence $H_{n}^{m} \bar{x} \varphi(\bar{x}, \bar{v})$ ) and we will use it without making mention of it. This observation is true also for the equicardinality interpretation of $Q^{m}$ which is defined by $A \vDash Q_{c}^{m} \bar{x} \varphi(\bar{x})$ iff $A \vDash$ $Q_{\alpha}^{m} \bar{x} \varphi(\bar{x})$, where $|A|=\aleph_{\alpha}$ (cf. [BK]).

Following [TU2], a formula $\varphi(x, \bar{v})$ is called graduated in $T$ if $Q_{0}^{1}$ is $\varphi$-definable in $T$ (i.e. if $\varphi(x, \bar{a})$ defines a finite set then this set has cardinality $<n$, where $n$ can be chosen universally for all parameters $\bar{a}$ ). A theory is graduated if all formulae are graduated in $T$. Now it is easily seen that a theory admits the elimination of $Q_{0}^{1}$ iff it is graduated [TU2].

It turns out that the following property introduced by Keisler is a good generalization of nongraduatedness. $\varphi(\bar{x}, \bar{v})$ has the finite cover property $($ f.c.p. $)$ if in some model $A$ of $T$ for arbitrarily large $n$ there exist $\bar{a}_{0}, \ldots, \bar{a}_{n-1}$ such that

$$
A \vDash \underset{j<n}{\&}\left(\exists \bar{x} \underset{i \neq j}{\&} \varphi\left(\bar{x}, \bar{a}_{i}\right)\right) \& \neg \exists \bar{x} \underset{i<n}{\&} \varphi\left(\bar{x}, \bar{a}_{i}\right) .
$$

Note that $\psi(x, \vec{u}) \leftrightharpoons(\varphi(x, \vec{v}) \& x \neq u)$, where $\bar{u}=\bar{v} \frown u$, has f.c.p. if $\varphi(x, \vec{v})$ is not graduated. By definition, $T$ has the f.c.p. if some formula $\varphi(x, \bar{v})$ has the f.c.p.

Feferman introduced another quantifier which is closely related to the f.c.p. Let $\varphi(x, z)$ be an $L$-formula. Then $\forall z\left(\varphi\left(x_{1}, z\right) \leftrightarrow \varphi\left(x_{2}, z\right)\right)$ determines an equivalence relation. We define $A \vDash Q_{\alpha}^{E} x z \varphi(x, z)$ if this equivalence relation has at least $\aleph_{\alpha}$ distinct equivalence classes on $A$ (cf. [FE, p. 129]).

We will use another version of this quantifier defined by $A \vDash E_{\alpha} x z \varphi(x, z)$ if $\varphi(x, z)$ is an equivalence relation with $\geqslant \boldsymbol{\aleph}_{\alpha}$ many equivalence classes on $A$.

Eliminability of $Q_{\alpha}^{E}$ is equivalent to eliminability of $E_{\alpha}$, since they are definable by each other:

$$
\begin{aligned}
& A \vDash E_{\alpha} x z \varphi(x, z) \leftrightarrow\left(Q_{\alpha}^{E} x z \varphi(x, z) \& e l(\varphi)\right), \\
& A \vDash Q_{\alpha}^{E} x z \varphi(x, z) \leftrightarrow E_{\alpha} x z(\forall v(\varphi(x, v) \leftrightarrow \varphi(z, v))),
\end{aligned}
$$

where $e l(\varphi)$ is a first order sentence which says " $\varphi(x, z)$ is an equivalence relation". Note that $Q_{\alpha}^{1}$ is definable using $E_{\alpha}$.

Having in mind the equivalence of definability and eliminability of the quantifier $E_{\alpha}$, we are able to give the following restatement of Shelah's f.c.p. theorem (cf. [SH, II, 4.4]):

THEOREM (THE F.C.P. THEOREM). Let $T$ be stable. Then the following are equivalent:

(i) $T$ has the f.c.p., i.e. some $\varphi(x, \bar{v})$ has the f.c.p.

(ii) $)_{m}$ Some $\varphi(\bar{x}, \bar{v})$ has the f.c.p., $l(\bar{x})=m$.

(iii) $E_{0}$ is not eliminable in $T .{ }^{1}$

Obviously, $E_{\alpha}$ is definable by $Q_{\alpha}^{2}$. Thus eliminability of $Q_{\alpha}^{2}$ implies eliminability of $E_{\alpha}$. Further, one can directly prove that a theory without the f.c.p. admits the

\footnotetext{
'One can introduce $E_{\alpha}^{m}$ also for $m$-tuples. Then (iii) $)_{m}$ is equivalent to the other conditions.
} 
elimination of all the $Q_{0}^{m}$. So, by the f.c.p. theorem, we get for $T$ a stable theory, $T$ does not have the f.c.p. iff $Q_{0}^{m}$ is eliminable in $T(m>1)$ iff $E_{0}$ is eliminable in $T^{2}$ This is Theorem 6 of [BK]. We proved it independently.

Note that an $\aleph_{1}$-categorical theory does not have the f.c.p. (Keisler's theorem), and all unstable theories do have it.

There are examples of unstable theories admitting the elimination of all $Q_{0}^{m}$ (e.g. $\aleph_{0}$-categorical theories; see also [BK]), but at present it is not known what class of unstable theories is determined by this property.

2. The two cardinal theorem. This section partially resembles $\$ 22$ of [SA]. So we will omit some proofs. First of all, let us prove some technical lemmas. Let $R, K$ be new unary predicate symbols, $\underline{c}$ a new constant symbol. Then $S(\underline{c}, \varphi(\bar{x}, \bar{a}))$ denotes the union of the following sets:

(1) $\left\{\forall \bar{v}\left(\bar{K}(\bar{v}) \rightarrow\left(F(\bar{v}) \leftrightarrow F^{K}(\bar{v})\right)\right): F(\bar{v}) \in L\right\} \cup T$;

(2) $\{\neg K(\underline{c})\}$;

(3) $\{\forall x(R(x) \rightarrow K(x)) \& \bar{K}(\bar{a})\}$;

(4) $\{\forall \bar{x}(\bar{R}(\bar{x}) \rightarrow \varphi(\bar{x}, \bar{a}))\}$;

(5) $\left\{\forall x_{0}\left(\forall x_{1} \ldots x_{m-1}\left(\&_{i=1}^{m-1} R\left(x_{i}\right) \rightarrow \&_{\bar{u} \in J} \varphi(\bar{u}, \bar{a})\right) \rightarrow R\left(x_{0}\right)\right)\right\}$, where $J=\left\{x_{0}, \ldots, x_{m-1}\right\}^{m}$.

LEMMA 2.1. $B$ is a model of $S(\underline{c}, \varphi(\bar{x}, \bar{a}))$ iff $B$ is a model of $T$, there is $A \prec B$ with a subset $Y$ (of $A)$ such that $A \neq B$, and $Y$ is maximally homogeneous for $\varphi(\bar{x}, \bar{a})$ in $B$, and $\bar{a} \in A$.

Proof. Let $B$ be a model of that set, $A$ the restriction of $B$ to $K(B), Y$ the set $R(B)$. Then $A \prec B, A \neq B, \bar{a} \in A \supseteq Y$ by (1)-(3) resp. (4) guarantees homogeneity, and (5) maximality of $Y$. Similarly the other direction. Q.E.D.

Now we will prove a series of analoga of lemmata from [SA, \$22]. Notice, a maximal homogeneous set for a formula $\varphi(x)$ (in one free variable) in $A$ is $\varphi(A)$ itself. Thus the maximal homogeneous sets for formulae in one free variable are exactly the definable sets. This is the point in the analogy mentioned above: We will extend the two cardinal theorem to formulae in $m$ free variables.

The next lemma is the appropriate analogon of [SA, 22.2].

Lemma 2.2. If $A \prec B, A \neq B, \bar{a} \cup Y \subseteq A, B$ countable, $b \in B-A, p$ a complete $n$-type over $\left\{a_{i}: i<k\right\} \subseteq A$, and $Y$ maximally homogeneous for $\varphi(\bar{x}, \vec{a})$ in $B$, then there are $A^{\prime}>A, B^{\prime}>B, Y^{\prime}$ with the same properties, but $p$ realized in $A^{\prime}$.

Proof. Let $S$ be the union of the following sets:

(6) $\operatorname{Th}(\langle B, B\rangle) \cup\{K(a): a \in A\}$;

(7) $(\operatorname{Th}(\langle A, A\rangle))^{K}$;

(8) $S(\underline{b}, \varphi(\bar{x}, \bar{a})$ ), where $b$ and $\bar{a}$ are as in the hypothesis (notice, $b$ occurs also in (6));

\footnotetext{
${ }^{2} E_{\alpha}^{m}$ is definable using $Q_{\alpha}^{*, 2, m}$ of $\left[\mathrm{BK}, \mathrm{p}\right.$. 7]. The eliminability of $E_{0}^{m}$ is also equivalent to $\neg$ f.c.p. in the stable case by Theorem 7 of [BK].
} 
(9) $\{F(\bar{c}) \& \bar{K}(\bar{c}): F(\bar{x}) \in p\}$, where $\bar{c}$ is an $n$-tuple of new constant symbols not occurring in $\left\{\underline{b}^{\prime}: b^{\prime} \in B\right\}$.

$S$ is consistent, as every finite subset is realized in $B$ for the interpretation $K(B)=A, R(B)=Y$. Take a countable model $B^{\prime}$ of $S, A^{\prime}$ the restriction of $B^{\prime}$ to $K\left(B^{\prime}\right), Y^{\prime}=R\left(B^{\prime}\right)$. Then $B^{\prime}>B$ by (6), $A^{\prime}>A$ by (6) and (7), $p$ is realized in $A^{\prime}$ by (9), $b \in B^{\prime}-A^{\prime}$ by (2) and (8). The rest follows by (8) and Lemma 2.1 . Q.E.D.

The next lemmata are the analoga of 22.3, 22.4 of [SA], resp. We omit the proofs, since one only has to put " $Y \subseteq N$ and $Y$ is maximally homogeneous for $\varphi(\bar{x}, \bar{a})$ in $M$ " instead of " $R(N)=R(M)$ " whenever $N \prec M$ and $N \neq M$ in the original proofs.

LeMMA 2.3. Let $A, B$, and $Y$ be as in the hypothesis of Lemma 2.2. Then there are $A^{\prime}, B^{\prime}, Y^{\prime}$ with the same properties such that $A^{\prime}>A, B^{\prime}>B, A^{\prime} \cong B^{\prime}, A^{\prime}$ and $B^{\prime}$ are countable homogeneous models.

This lemma tells us that w.l.o.g. we can assume $A$ and $B$ to be isomorphic and homogeneous models in the situation of the next lemma.

Lemma 2.4. Let $A, B, Y$ be as in Lemma 2.3. Then there are a model $C>B$ and $a$ maximal homogeneous set $Y^{\prime}$ for $\varphi(\bar{x}, \bar{a})$ in $C$ such that $|C|=\aleph_{1}$ and $\left|Y^{\prime}\right| \leqslant \aleph_{0}$. If $|Y|=\aleph_{0}$, then one can assume $\left|Y^{\prime}\right|=\aleph_{0}$.

Now we introduce a generalization of the notion of a Vaughtian pair.

DEFINITION 2.5. (i) $(A, B)$ is called a generalized Vaughtian pair for $\varphi(\bar{x}, \bar{a})$ if $A \prec B, A \neq B, \bar{a} \in A$, and there is an infinite subset $Y$ of $A$ which is maximally homogeneous for $\varphi(\bar{x}, \bar{a})$ in $B$.

(ii) $(A, B)$ is called a generalized Vaughtian pair (of index $m)$ if $(A, B)$ is a generalized Vaughtian pair for a formula $\varphi(\bar{x}, \bar{a})$ with $\bar{a} \in A$ (and $l(\bar{x})=m$ ).

(iii) Let $\varphi(\bar{x})$ be a fixed formula of $L . T$ is said to admit a pair of cardinals $(\mu, \chi)$ if $T$ has a model $B$ of cardinality $\mu$ and there is a subset $Y$ of $B$ of cardinality $\chi$ such that $Y$ is maximally homogeneous for $\varphi$ in $B$.

Notice, a generalized Vaughtian pair of index 1 is a usual Vaughtian pair.

LEMMA 2.6. If there is a generalized Vaughtian pair for a certain formula, then there is a countable one for that formula.

Proof. By the hypothesis and Lemma 2.1 the set $S(\underline{c}, \varphi(\bar{x}, \bar{a})) \cup\left\{R\left(\underline{c}_{i}\right) \& \underline{c}_{i} \neq \underline{c}_{j}\right.$ : $i<j<\omega\}$ is consistent. Take a countable model and define a generalized Vaughtian pair as in Lemma 2.1. Q.E.D.

Definition 2.7. (i) A model $A$ is called $\varphi$-regular if any infinite set which is maximally homogeneous for $\varphi$ in $A$ has the cardinality $|A| . A$ is called $\varphi$-singular if it is not $\varphi$-regular.

(ii) A model $A$ is called $m$-regular if it is regular for all formulae in $m$ free variables.

(iii) A model $A$ is called regular if it is $m$-regular for all $m$. 
(iv) A theory is called $\varphi$-regular if all models of $T$ are $\varphi$-regular. Similarly for $m$-regularity and regularity of $T$.

Now we are able to prove the main theorem of this section.

THEOREM 2.8 (THE TWO CARDINAL THEOREM FOR HOMOGENEOUS SETS). (i) $T$ has no Vaughtian pairs for $\varphi(\bar{x}, \bar{a})$ iff any model containing $\bar{a}$ is $\varphi$-regular.

(ii) $T$ has no Vaughtian pairs (of index $m$ ) iff any model is $\varphi$-regular for all formulae $\varphi$ with ( $m$ free variables and) parameters from that model.

(iii) If $T$ admits $(\mu, \chi)$ for $\mu>\chi \geqslant \boldsymbol{\aleph}_{0}, T$ admits $\left(\boldsymbol{\aleph}_{1}, \boldsymbol{\aleph}_{0}\right)$.

Proof. (i) $\Rightarrow$. Suppose to the contrary there is an infinite set $Y \subseteq A$ of cardinality less than $|A|$, such that $Y$ is maximally homogeneous for $\varphi(\bar{x}, \bar{a})$ in $A$. Let $B$ be an elementary submodel of $A$ extending $Y$ which has the same cardinality as $Y$. Then $B \neq A$ and thus $(B, A)$ is a generalized Vaughtian pair for $\varphi(\bar{x}, \bar{a})$.

$\Leftarrow$. Suppose $(A, B)$ is a generalized Vaughtian pair for $\varphi(\bar{x}, \vec{a})$. By Lemma 2.6, w.l.o.g. $B$ is countable. By Lemma 2.4 , there is a model $C>B$ and a maximal homogeneous set $Y$ for $\varphi(\bar{x}, \bar{a})$ in $C$ such that $|C|=\aleph_{1}$ and $|Y|=\aleph_{0}$, contradicting the hypothesis.

(ii) follows from (i).

(iii) Suppose $B \vDash T,|B|=\mu, Y \subseteq B$ is a maximal homogeneous set for $\varphi(\bar{x}, \vec{a})$ in $B,|Y|=\chi$. By the first half of (i), there is a generalized Vaughtian pair $(A, B)$ for $\varphi$ which is countable by Lemma 2.6. Now, by Lemma $2.4, T$ admits $\left(\aleph_{1}, \aleph_{0}\right)$. Q.E.D.

The reader will not be very surprised by the following.

THEOREM 2.9. Any model of an $\aleph_{1}$-categorical theory is regular (or, equivalently, an $\aleph_{1}$-categorical theory has no generalized Vaughtian pairs).

Proof. By definition, a singular model is uncountable. Now the assertion follows from the next lemma. Q.E.D.

LEMMA 2.10. Any saturated model is regular.

Proof. Let $A$ be a singular model which is saturated, $Y \subseteq A$ a maximal homogeneous set for a formula in $A$ which has infinite cardinality less than $|A|$. Let $p(x)$ be the type saying " $x \notin Y$ and $\{x\} \cup Y$ is homogeneous for that formula". Then $p$ is realized in $A$, contradicting the maximality of $Y$. Q.E.D.

The class of regular theories seems to us to be of interest for further investigation. We close this section with the following observation.

LEMMA 2.11. If all models of $T$ of power $\aleph_{1}$ are $\varphi$-regular, then $T$ is $\varphi$-regular.

Proof. By Theorem 2.8(iii). Q.E.D.

COROLlaRy 2.12. If all models of $T$ of power $\aleph_{1}$ are regular, $T$ is regular.

3. The elimination of Malitz quantifiers. Now we are able to give sufficient conditions for the eliminability of the quantifiers in terms of regularity introduced in the preceding section. 
THEOREM 3.1. (i) If every model of $T$ is $\varphi$-regular $(\varphi=\varphi(\bar{x}, \bar{v}), l(\bar{x})=m)$, then the quantifier $Q_{c}^{m}$, hence also $Q_{\alpha}^{m}$ for $\alpha \geqslant 0$, is $\varphi$-eliminable in $T$.

(ii) If $T$ is $m$-regular, or, equivalently, $T$ has no generalized Vaughtian pairs of index $m$, all the quantifiers $Q_{c}^{m}$ and $Q_{\alpha}^{m}$ are eliminable in $T(\alpha \geqslant 0)$.

(iii) If $T$ is regular, or, equivalently, $T$ has no generalized Vaughtian pairs, the quantifiers $Q_{c}^{m}$ and $Q_{\alpha}^{m}$ are eliminable in $T$ for all $m \geqslant 1$ and $\alpha \geqslant 0$.

Proof. (i) implies (ii) and (iii). First of all we prove

Claim. If every model of $T$ is $\varphi$-regular, then $Q_{0}^{m}$ is $\varphi$-eliminable in $T(\varphi=$ $\varphi(\bar{x}, \bar{v}), l(\bar{x})=m)$.

Proof of THE Claim. For the contrary, suppose $Q_{0}^{m}$ is not $\varphi$-eliminable in $T$. Then for all $n$ there is a model $A_{n}$ of $T$ and $\bar{a}_{n} \in A_{n}$ such that $A_{n}$ has a maximal homogeneous set for $\varphi(\bar{x}, \bar{a})$ which has finite cardinality $\geqslant n$. Then every finite subset of $S(\underline{c}, \varphi(\bar{x}, \bar{a})) \cup\left\{R\left(\underline{c}_{i}\right) \& \underline{c}_{i} \neq \underline{c}_{j}: i<j<\omega\right\}$ (cf. §2) is consistent (w.l.o.g. $A_{n}$ is uncountable; put $K\left(A_{n}\right)=B$, where $B$ is a countable elementary submodel of $A_{n}$ containing the finite maximal homogeneous set).

By Lemma 2.1, every model yields a Vaughtian pair for $\varphi(\bar{x}, \bar{a})$. Thus, by the two cardinal theorem, there is a model which is not $\varphi$-regular, whence the claim is proved.

Now suppose $Q_{c}^{m}$ is not $\varphi$-eliminable in $T$. Then there is a model $B$ of $T$ with $\bar{a} \in B$ and

$$
B \vDash H_{n}^{m} \bar{x} \varphi(\bar{x}, \bar{a}) \& \neg Q_{c}^{m} \bar{x} \varphi(\bar{x}, \bar{a}),
$$

where $n$ is chosen to satisfy

$$
A \vDash \forall \bar{v}\left(H_{n}^{m} \bar{x} \varphi(\bar{x}, \bar{v}) \rightarrow Q_{0}^{m} \bar{x} \varphi(\bar{x}, \bar{v})\right)
$$

for all $A \vDash T$ using the claim. So

$$
B \vDash Q_{0}^{m} \bar{x} \varphi(\bar{x}, \bar{a}) \& \neg Q_{c}^{m} \bar{x} \varphi(\bar{x}, \bar{a}),
$$

whence $B$ is a $\varphi$-singular model, contradicting the hypothesis. Q.E.D.

The converse seems hard to come by, since a formula with more than one free variable $(m>1)$ can have maximal homogeneous sets of different cardinality. However, for $m=1$ the following were already proved in [TU2].

COROLlARY 3.2. The following properties are equivalent for a theory $T$ :

(i) $Q_{1}^{1}$ is eliminable;

(ii) $Q_{c}{ }_{c}$ is eliminable;

(iii) for all $\alpha \geqslant 0, Q_{\alpha}^{1}$ is eliminable;

(iv) $T$ has no (usual) Vaughtian pairs, or, equivalently, $T$ is 1-regular.

Proof. Remember, the usual Vaughtian pairs are exactly the generalized Vaughtian pairs of index 1. Now one direction is Theorem 3.1(ii). For the other, note that a formula in one free variable has only one maximal homogeneous set. Thus, if $Q_{1}^{1}$ is eliminable, every model of power $\aleph_{1}$ is 1-regular. By Lemma $2.11, T$ is regular. Q.E.D.

Similarly one can prove

Corollaky 3.3. If $Q_{\alpha}^{1}$ is eliminable in $T$ for some $\alpha \geqslant 0, Q_{0}^{1}$ is eliminable in $T$. 
So, eliminability of $Q_{1}^{1}$ is the strongest notion, that of $Q_{0}^{1}$ the weakest, whereas eliminability of the $Q_{\alpha}^{1}$ 's is between them.

In case of stability an analogon of Morley's categoricity theorem is true (cf. [TU2]):

Proposition 3.4. For $T$ a stable theory, $T$ admits the elimination of $Q_{1}^{1}$ iff $T$ admits the elimination of $Q_{\alpha}^{1}$ for some $\alpha \geqslant 1$.

We do not know what can happen if $T$ is unstable. The general case presumably depends on set theory. Also we do not know what is the matter in case $m>1$. Generally, there are two kinds of questions concerning the relative strength of eliminability of Malitz quantifiers:

What is the relation between the eliminability of

(a) $Q_{\alpha}^{m}$ and $Q_{\alpha}^{n}\left(Q_{c}^{m}\right.$ and $\left.Q_{c}^{n}\right)$ and of

(b) $Q_{\alpha}^{m}$ and $Q_{\beta}^{m}$.

For $\alpha=0$, there is an answer to (a) for stable theories:

Eliminability of $Q_{0}^{2}$ is equivalent to that of $Q_{0}^{m}(m>1)$; cf. $\S 1$ or [BK].

For $m=1$, the present section provides some answers to (b) which are complete when stability is assumed.

The theory RCF of real closed fields is an example of an unstable complete theory which admits elimination of $Q_{1}^{1}$, but not of $Q_{1}^{2}$. (RCF has no (usual) Vaughtian pairs; using $Q_{1}^{2}$ one can define the sentence "there is an uncountable discrete subset cofinal in the field" which is consistent with RCF, but not true in R; similarly, RCF admits elimination of $Q_{0}^{1}$, but not of $Q_{0}^{2}$.)

Perhaps from [GA] one can obtain examples of theories admitting the elimination of $Q_{\alpha}^{m}$, but not of $Q_{\alpha}^{m+1}$ for all $\alpha>0$ and $m>1$ (for this one has to show that not only the structures $\mathfrak{A}$ and $\mathfrak{B}$, but the theory $\mathrm{Th}(\mathfrak{A})$ admits elimination of $Q_{\alpha}^{m}$; cf. [GA, Lemma 2]).

Let us conclude this section with a theorem which was also proved by Baldwin and Kueker [BK, Theorem 9].

THEOREM 3.5. An $\aleph_{1}$-categorical theory admits the elimination of $Q_{c}^{m}$ and $Q_{\alpha}^{m}$ for all $m \geqslant 1$ and $\alpha \geqslant 0$.

Proof. By Theorem 3.1(iii) and 2.9. Q.E.D.

4. On a question of Baldwin and Kueker. In the present section we will show that an example of Makowsky, which goes back to R. Robinson, is in fact a counterexample to the following question of Baldwin and Kueker [BK, Question 4]:

For $T$ a theory in a finite language, if $T$ admits the elimination of $Q_{c}^{m}$ for all $m \geqslant 1$, must $T$ be $\aleph_{1}$-categorical (or, equivalently, $\omega$-stable)?

While investigating the elimination of $Q_{1}^{m}$ the second author had the same question (however for $Q_{1}^{m}$ ), whereas the first author suggested Makowsky's example for a negative answer.

We would like to thank H. Herre who brought to our attention that [MA] provides an example of a superstable theory without (usual) Vaughtian pairs which is finitely axiomatizable. 
Now let us introduce the example. For details and proofs we refer to [MA]. Let $G=F / R$ be a group with $F, R$ free countably generated groups. Let $A_{G}$ be the graph of the group $G$, i.e. $A_{G}=\left\langle A, f_{i}: i \in I\right\rangle$, where $\left\{f_{i}: i \in I\right\}$ is a set of generators of $F, f_{i}$ is a unary function symbol $(i \in I)$, and for all $a \in A$, $\underline{f}_{i_{1}} \cdots f_{i_{k}}(a)=a$ iff $f_{i_{1}} \cdot \ldots \cdot f_{i_{k}} \in R$.

We assume that for all $i \in I$ there is a $j \in I$ such that $f_{i} f_{j}(a)=f_{j} f_{i}(a)=a$ for all $a \in A$. In other words, we assume that the set of generators of $\bar{F}$ is closed under taking the inverse.

Let $P_{1}, \ldots, P_{n}$ be unary predicates on $A_{G}$. From now on, $T=$ $\operatorname{Th}\left(A_{G}, P_{1}, \ldots, P_{n}\right)$, and $T_{G}=\operatorname{Th}\left(A_{G}\right) . T$ is model complete and superstable. Following [MA], call a substructure of a model of $T$ which is generated by a single element a component of that model. Denote by $C(a)$ the component generated by $a$. Then every component is countable and every model of $T$ splits into disjoint components which are all isomorphic relative to $L_{G}$, the language of $T_{G}$.

Makowsky proved for universal $T$ that $T$ is $\aleph_{1}$-categorical iff it is $\omega$-stable. This follows also from Corollary 4.4. Our aim is to show that $T$ has no generalized Vaughtian pairs if $T$ is universal.

From the model completeness of $T$ we obtain the following.

LEMMA 4.1. If $T$ is universal, $T$ admits the elimination of (usual) quantifiers.

Considering various examples of graphs of groups the next lemma becomes evident.

LEMMA 4.2. $A_{G}$ is totally homogeneous, i.e. for all $a, b \in A_{G}$ there is an $L_{G^{-}}$ automorphism $F$ of $A_{G}$ mapping $a$ onto $b$.

Proof. Let $1 \in A$ correspond to the unity of the group $G$. There are representations of $a$ and $b$ through 1 , since $A_{G}$ is connected: $a=f_{i_{1}} \cdots f_{i_{m}}(1), b=$ $f_{j_{1}} \cdots f_{j_{n}}(1)$. Now we define $F$ as follows: for $c=f_{k_{1}} \cdots f_{k_{l}}(1)$ an arbitrary element of $A$, set $F(c)=f_{k_{1}} \cdots f_{k_{1}} f_{i_{m}}^{-1} \cdots f_{i_{1}}^{-1} f_{j_{1}} \cdots f_{j_{n}}(\overline{1})$. Clearly, $F(a)=b$ and $f(F(c))$ $=F(f(c))$ for $\left.f \in f_{i}: i \in \bar{E}\right\}$, whence $\bar{F}$ is a homomorphism. Also it is not hard to verify that $F$ is a $1-1$ map, since

$$
A_{g} \vDash \forall x\left(\underline{f}_{k_{1}} \cdots \underline{f}_{k_{l}}(x)=x\right) \leftrightarrow \exists x\left(\underline{f}_{k_{1}} \cdots \underline{f}_{k_{l}}(x)=x\right) \text {. Q.E.D. }
$$

The following lemma is the main one.

LemMA 4.3. Let $B \vDash T, A \prec B, A \neq B, Y \subseteq A, Y$ homogeneous for $\varphi(\bar{x}, \bar{a})$, $\bar{a} \in A$. If $T$ is universal, $Y$ is not maximally homogeneous for $\varphi$ in $B$.

Proof. $Y \cup\{c\}$ is homogeneous for $\varphi$ in $C$ for some proper extension $C>A$ and some $c \in C-A$. W.l.o.g. $C=A \cup C(c)$.

By Lemma 4.1, there are $G_{i}(\bar{x}, \bar{v}), R_{i}(\bar{x})$, and $F_{i}(\bar{v})(i=1, \ldots, k)$ such that

$$
T \vdash \varphi(\bar{x}, \bar{v}) \leftrightarrow \bigvee_{i=1}^{k}\left(G_{i}(\bar{x}, \bar{v}) \& R_{i}(\bar{x}) \& F_{i}(\bar{v})\right)
$$

and $G_{i} \in L_{G}, R_{i}(\bar{x})$ and $F_{i}(\bar{v})$ are conjunctions of the $P_{j}^{\prime}$ 's and their negations $(i=1, \ldots, k)$. (Generally, there can be $L_{G}$-terms in $R_{i}, F_{i}$; the proof remains the 
same.) In $B$ there is a component $C(b)$ elementarily equivalent to $C(c)$, since both are models of $T$ for $T$ is universal. W.l.o.g. $B=A \cup C(b)$. In $C(b)$ there is an element which has the same " $\left\{\boldsymbol{P}_{1}, \ldots, \boldsymbol{P}_{n}\right\}$-type" as has $c$. Therefore without loss we can assume that $b$ has that property.

We claim $Y \cup\{b\}$ is homogeneous for $\varphi$. For this, let $\bar{y} \in(Y \cup\{b\})^{m}$ and $\bar{y}^{\prime}$ be the sequence obtained from $\bar{y}$ by substituting $b$ by $c$.

We have to show $B \vDash \varphi(\bar{y}, \bar{a})$. $C(c)$ and $C(b)$ are models of $T$, in particular, both are models of $T_{G}$, whence they are isomorphic to $A_{G}$ as $L_{G}$-structures. Using Lemma 4.2 we choose an $L_{G}$-isomorphism $F: C \cong B$ which maps $c$ onto $b$ and is the identity map on $A$. Thus, $B \vDash G_{i}(\bar{y}, \vec{a})$ iff $C \vDash G_{i}\left(\bar{y}^{\prime}, \vec{a}\right)$ for all $i$. Also, $B \vDash R_{i}(\vec{y})$ iff $C \vDash R_{i}\left(\bar{y}^{\prime}\right)$, since by construction $\bar{y}$ and $\bar{y}^{\prime}$ have the same $\left\{P_{1}, \ldots, P_{n}\right\}$-type. So we have $C \vDash \varphi\left(\bar{y}^{\prime}, \bar{a}\right)$ iff $B \vDash \varphi(\bar{y}, \bar{a})$. But $C \vDash \varphi\left(\bar{y}^{\prime}, \bar{a}\right)$ for $Y \cup\{c\}$ is homogeneous for $\varphi$, whence the result. Q.E.D.

COROllary 4.4. Let $T$ be universal. Then:

(i) $T$ is regular, or, equivalently, $T$ has no generalized Vaughtian pairs.

(ii) $T$ admits the elimination of $Q_{c}^{m}$ and $Q_{\alpha}^{m}$ for all $m \geqslant 1$ and $\alpha \geqslant 0$.

Proof. (i) follows from Lemma 4.3, (ii) from (i) and Theorem 3.1(iii). Q.E.D.

Using a domino of $\mathrm{R}$. Robinson, Makowsky showed that there is a universal theory $T=\operatorname{Th}\left(\left\langle A_{G}, P_{1}, \ldots, P_{n}\right\rangle\right)$ in a finite language which is finitely axiomatizable and not $\omega$-stable (cf. [MA, p. 200]). Thus we obtain the following theorem which yields the desired answer to the question mentioned in the beginning of this section.

THEOREM 4.5. There is a theory $T$ in a finite language such that:

(i) $T$ is superstable, but not $\omega$-stable;

(ii) $T$ has no generalized Vaughtian pairs;

(iii) $T$ admits the elimination of $Q_{c}^{m}$ and $Q_{\alpha}^{m}$ for all $m \geqslant 1$ and $\alpha \geqslant 0$.

(iv) $T$ is finitely axiomatizable.

APPENDIX. One can define homogeneity and regularity for $\psi\left(\bar{x}_{1}, \ldots, \bar{x}_{m}, \bar{v}\right)$ and extend 2.8 to that case. Then 3.1 extends to $Q_{\alpha}^{*, m, n}$ of [BK]. The converse of 3.1 holds if all infinite maximal homogeneous sets have the same cardinality. Of course, this is true for an equivalence relation (a maximal homogeneous set is a set of representatives of each class). Thus, eliminability of $E_{1}$ implies that of $E_{0}$. Now, by the f.c.p. theorem, for $T$ a stable theory, if $T$ admits elimination of $Q_{1}^{2}, T$ does not have the f.c.p., whence all $Q_{0}^{m}$ are eliminable.

ADDED IN PROOF. We have proved the converse of Theorem 3.1(iii) for stable theories $T$. This will appear in a subsequent paper.

\section{REFERENCES}

[BK] J. T. Baldwin and D. W. Kueker, Ramsey quantifiers and the finite cover property, Pacific J. Math. 90 (1980), 11-19.

[FE] S. Feferman, Two notes on abstract model theory. II, Fund. Math. 89 (1975), 111-130.

[GA] S. Garavaglia, Relative strength of Malitz quantifiers (preprint).

[MA] J. A. Makowsky, On some conjectures connected with complete sentences, Fund. Math. 81 (1974), 193-202. 
[MM] M. Magidor and J. Malitz, Compact extensions of L(Q), Ann. Math. Logic 11 (1977), 217-261. [SA] G. E. Sacks, Saturated model theory, Benjamin, New York, 1972.

[SH] S. Shelah, Classification theory and the number of non-isomorphic models, North-Holland, Amsterdam, 1978.

[TU1] P. Tuschik, $\aleph_{1}$-Kategorizität, Dissertation (A), Humboldt-Univ., Berlin, 1975.

[TU2] _ Elimination of cardinality quantifiers (contributed paper at the 4th Bierutowice Conf., Karpacz, Poland, September 1979).

AKademie der Wissenschaften DER DDR, Institut für Mathematik, 1080 Berlin, German Democratic RePUblic

Sektion Mathematik, Humboldt-Universität zu Berlin, 1086 Berlin, German Demokratik REPUBLIK 